London has directed attention to the fact that an ideal Bose-Einstein gas shows a transition point, which lies at $3 \cdot 15^{\circ} \mathrm{K}$. for a gas of a density and a molecular weight of liquid helium. MIoreover, below this transition point the gas allows also a division of the molecules in those condensed in the lowest energy state, and others having a kinetic energy.

However, it can scarcely be expected that the velocity distribution of the moving particles in liquid helium can be identified with that of an ideal BoseEinstein gas, so that it is not surprising that the specific heat of such an ideal gas" does not agree with that of liquid helium. The specific heat should increase $T^{3 / 2}$, whereas experiments show an increase with, at least, $T^{3} .5$

This difficulty can be eliminated by the assumption of an 'excitation-energy', which must bo added to a condensed particle before it can participate in the velocity distribution of the moving ones. The specific heat increases then exponentially at low temperatures. In this way a better representation of the anomaly in the specific heat is obtained.

The existence of such an 'excitation-energy' can be explained on similar lines as the disorder energy in Frōhlich's theory of liquid helium ${ }^{6}$. On the other hand, it may also bo derived from the gas model, as a consequence of the theory of a non-ideal BoseEinstein gas below its transition temperature.

Here the excitation energy is due to the effect of exchange, which gives rise to a different interaction when molecules are in the same and when they are in different states. This effect leads to important corrections in the specific heat below the transition temperature, as then many particles will occupy the same, namely, the 'condensed' state.

A more detailed discussion will be published in Physica.

A. Michels.

A. I3IJL.

J. DF BOER.

Van der Waals Laboratorium,

Amsterdam.

July 31 .

'Allen, J. F., and Jones, II., Nature, 141, 243 (1938).

2 Michels, A., Bijl, A., and de Boer, J., Physica, 5, 121 (1938).

Tisza, L., Natcre, 141, 913 (1933).

' London, F., NATtre, 141, 613 (1033).

' Keesom, W. H., and Kecsom, A. P., Physica, 2, 557 (1935).

- Fröhlich, H., Physica, 4, 639 (1937).

\section{Specific Heat of a Substance showing Spontaneous} Electric Polarization

VARIous authors ${ }^{1}$ have investigated the specific heat of Rochelle salt, the best-known example of substances showing spontaneous electric polarization, but the results obtained so far have been somewhat contradictory. In some of the experiments a small anomaly at the Curie point (the upper limit of the Rochello olectric temperature region) was observed. Very little is, however, known about any thermal effects at the lower limit of the Rochelle-electric region. This temperature has sometimes been called the 'lower Curie point', and it has been suggested" that it should be marked by an anomaly in the specific heat.

We have now determined the specific heat of potassium dihydrogen phosphate whichshowsRochelle electricity in the temperature region between $75^{\circ}$ and $122^{\circ} \mathrm{K}^{3}$. The result of this investigation is given in the accompanying graph. It is evident from our measurements that there is no trace of any anomaly at the 'lower Curie point'. This means probably that the apparent disappearance of spontaneous electric polarization towards lower temperatures is due to a 'freezing-in' of the dipoles; that is, their inability to orientate themselves below a certain temperature owing to lack of thermal motion in the lattice. It seems, therefore, misleading to describe this temperature as a 'lower Curio point'.

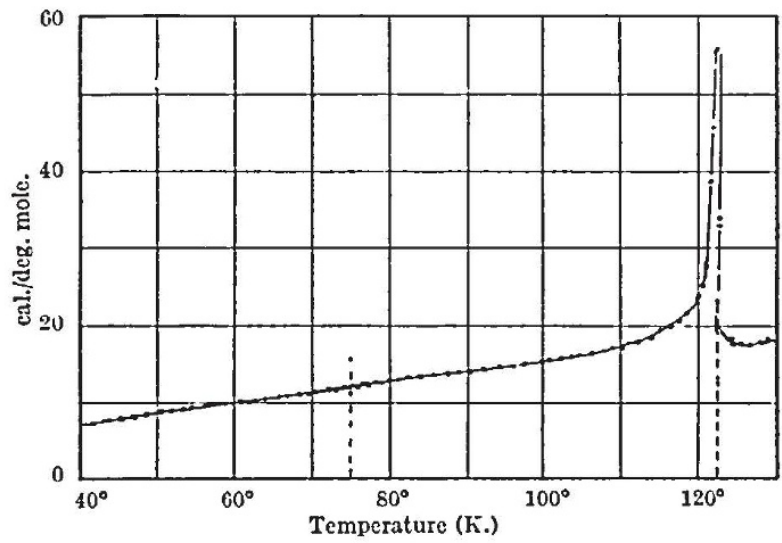

A strong anomaly was observed at the Curie point $\left(122^{\circ} \mathrm{K}\right.$.). Owing to the very gradual rise of the specific heat at tho onset of this anomaly, an accurate ovaluation of its energy content seems difficult. While these experiments were in progress, measurements of the specific heat at the Curie point were reported by Bantle and Scherrer ${ }^{4}$ and Stephenson and Hooley ${ }^{5}$. Whereas our values in this temperature region agree well with the results of the latter authors, they differ considerably from those of Bantle and Scherrer. These authors observe the anomaly at $114^{\circ} \mathrm{K}$. and give much higher values for the specific heat.

A full report of our experiments will bo published elsewhere.

J. Mendelssohn.

Clarendon Laboratory, I. Mendelssohn.

Oxford.

August 18.

Kobeko and Nelidow, Soxj. Phys, 1, 382 (1932). Rusterholz, Helo. phys. Acta, 8, 39 '(1935). Wilison, Phys. Rex., 54, 1103 (1938). Hicks and Hooley, J. Amer. Chem. Soc., 6), 2994 (1938). : Staub, Naturiciss., 43, 728 (1935).

${ }^{3}$ Busch, Helv. phys. Acta, 11, 269 (1938).

- Bantle and Scherrer, NATURE, 142, 950 (1939).

stephenson and Hooley, Phys. Rev., 53, 121 (1939).

\section{A Particular Mode of Fission of the Uranium Nucleus}

As has been reported by one of $\mathrm{us}^{1}$, uranium fission products can be collected by the recoil method many hours after the end of the irradiation of uranium by slow neutrons. This phenomenon can be explained in the simplest way by the assumption that one or more of the fission products are radioactive gases, of more or less long life and coming somewhere in the middle of the $\beta$-transmutation chain. To test this assumption we have performed the following experi. ments.

About $20 \mathrm{gm}$. of sodium pyrouranate placed in a glass tube between two cotton-wool plugs was irradiated by slow neutrons from a radio-thorium 